\title{
Ocular findings and ocular graft-versus-host disease after allogeneic stem cell transplantation without total body irradiation
}

\author{
Meeta Pathak ${ }^{1,2} \cdot$ Phoi Phoi Diep ${ }^{2,3} \cdot$ Xiaoran Lai $^{4} \cdot$ Lorentz Brinch $^{5} \cdot$ Ellen Ruud $^{2,3} \cdot$ Liv Drolsum ${ }^{1,2}$ \\ Received: 19 September 2017 / Revised: 10 December 2017 / Accepted: 23 December 2017 / Published online: 30 January 2018 \\ (c) The Author(s) 2018. This article is published with open access
}

\begin{abstract}
Patients treated with allogeneic stem cell transplantation (allo-SCT) often develop ocular complications. To investigate the ocular findings in young long-term survivors after allo-SCT without TBI, we examined 96 patients more than 5 years after transplantation. All patients were under 30 years of age at transplantation. The mean follow-up time was 16.8 years (range 6.0-26.1 years). The study was a part of the Norwegian Allo Survivorship Study investigating health impairments in young survivors after allo-SCT. Ophthalmological examination included visual acuity, tear break-up time, corneal fluorescein staining, Schirmer I test, tear film osmolarity, biomicroscopy and dilated ophthalmoscopy. In patients with known systemic chronic GVHD (cGVHD), ocular GVHD (oGVHD) diagnosed by clinical examination was compared with diagnosis using National Institutes of Health (NIH) or International Chronic Ocular Graft-vs-Host-Disease (ICCGVHD) Consensus Group criteria. We diagnosed dry eye disease (DED) in 52 patients (54\%), cataract in 3 patients (3\%) and retinopathy in 1 patient (1\%). Systemic cGVHD was a risk factor for DED (OR 4.40, CI 1.33-14.56, $p=0.02$ ). Comparison of diagnostic criteria suggests that the more stringent ICCGVHD criteria can better differentiate DED from oGVHD after allo-SCT as compared with the NIH criteria.
\end{abstract}

\section{Introduction}

Haematopoietic stem cell transplantation (SCT) is an established and potentially curative treatment modality for various malignant and non-malignant haematologic diseases. At present, $\sim 110-120$ patients are treated with allogeneic SCT (allo-SCT) each year in Norway, of whom 20-30 patients are younger than 18 years of age at the time of transplantation.

Unfortunately, patients treated with allo-SCT often develop graft-versus-host disease (GVHD), a potentially

Meeta Pathak

meeta.pathak@medisin.uio.no

1 Center for Eye Research, Department of Ophthalmology, Oslo University Hospital, Oslo, Norway

2 University of Oslo, Oslo, Norway

3 Children and Adolescent's Division, Oslo University Hospital, Rikshospitalet, Oslo, Norway

4 Department of Biostatistics, University of Oslo, Oslo, Norway

5 Department of Haematology, Oslo University Hospital, Rikshospitalet, Oslo, Norway life-threatening multi-organ systemic disease associated with significant morbidity and mortality [1]. Ocular GVHD (oGVHD) affects $40-60 \%$ of patients treated with allo-SCT [2-5]. Although oGVHD may be the only manifestation of GVHD, it is more frequently seen together with other systemic manifestations and 50-90\% of patients with systemic GVHD also have oGVHD [6, 7].

Typically, oGVHD involves the anterior segment of the eye, including the lid, lacrimal gland, conjunctiva and cornea. Clinically, the condition manifests primarily as dry eye disease (DED), with keratoconjunctivitis sicca (KCS), and resembles the clinical findings seen in other immunologically mediated inflammatory diseases of the ocular surface. DED is defined as a 'disorder of the tear film due to tear deficiency or excessive evaporation, which causes damage to the interpalpebral ocular surface and is associated with symptoms of ocular discomfort' [8]. Although DED can occur without keratitis, the terms DED and KCS are often used interchangeably [9]. Patients with DED usually experience symptoms such as redness, photophobia, foreign body sensation, excessive tearing, discharge, blurring of vision and pain [10]. The ocular surface affliction is irreversible in many cases and patients often experience a significant reduction in quality of life. Although rare, 
posterior segment involvement includes ischemic microvascular retinopathy, posterior scleritis, choroidal thickening and serous detachment $[11,12]$, which may significantly impair vision. Early detection, diagnosis and treatment are crucial to reduce morbidity and prevent blindness for longterm survivors.

There is no current international consensus on optimal criteria for the diagnosis of oGVHD [7, 13, 14] and clinical evaluation by an ophthalmologist is considered to be a historical gold standard $[9,15,16]$. Lack of pathognomonic symptoms and clinical findings make oGVHD diagnosis challenging. Various objective diagnostic tests may assist the clinical assessment of DED, including Schirmer's test, tear break-up time (TBUT), corneal and conjunctival staining and tear film osmolarity [13, 17]. However, differences in correlation to disease severity and varying, or even conflicting, results impose obvious limitations on the reliability of such tests alone $[17,18]$. Thus, test results must be considered simultaneously and in correlation with the patients subjective symptoms for a clinical diagnosis of DED and oGVHD.

In contrast to the protocol in many other countries, the conditioning regimen prior to allo-SCT in Norway has been based on myeloablative chemotherapy and has usually not included total body irradiation (TBI), with potentially different late effects compared with TBI-based conditioning regimens. The main aim of our study was to investigate the ocular findings and to identify possible risk factors for ocular complications in young patients (aged $\leq 30$ years at transplantation) more than 5 years after allo-SCT without TBI. The secondary aim of the study was to compare oGVHD diagnosis based on diagnostic criteria proposed by the U.S. National Institutes of Health (NIH) [19] and the International Chronic Ocular Graft-vs-Host-Disease (ICCGVHD) Consensus Group [14].

\section{Materials and methods}

The study was a cross-sectional, non-comparative clinical study of patients with leukemias, benign haematological diseases, immunodeficiencies or metabolic diseases treated with allo-SCT at Oslo University Hospital, Rikshospitalet, during the period April 1983 to August 2008. It was a part of the Norwegian Allo Survivorship Study (AlloSS-young) investigating health impairments in young survivors after allo-SCT. The following criteria were used for inclusion: all patients were alive and older than 16 years at study start (1 August 2014); all patients were aged $\leq 30$ years at transplantation; minimum time since transplantation was 5 years. Exclusion criteria included diagnosis of mucopolysaccaridosis type 1 (Mb. Hurler). A total of 157 eligible patients were invited to participate in the study, of which 103 patients $(69 \%)$ accepted. These patients were clinically evaluated by an experienced haematologist and by an ophthalmologist between August 2014 and January 2016. Seven patients had been treated with TBI and were subsequently excluded from analysis. Thus, a total of 96 patients were included in the study analysis. Of the 54 nonparticipating patients, 16 declined due to lack of time and health problems (one patient died), whereas 38 patients did not respond. Compared with the patients not included (declined or excluded), the study participants included more female patients $(54 \%$ vs $33 \%, p<0.01)$. There were no other statistically significant differences between the groups with regard to age at transplantation, time from transplantation to study start and benign or malignant diagnosis. Research approval was obtained from the Regional Committees for Medical and Health Research Ethics. Written informed consent was obtained from all the patients. The study was conducted in accordance with the Declaration of Helsinki.

\section{Study procedure}

Diagnosis of DED was based on patient symptoms, clinical findings of a comprehensive ocular examination and the results of diagnostic tests, which were performed according to the recommendations of the Dry Eye Work Shop [13]. All patients $(n=96)$ underwent a comprehensive ophthalmologic examination. Contact lenses were removed at least $30 \mathrm{~min}$ before measurements. History of decrease in vision, dryness, photophobia, foreign body sensation, irritation, redness, burning, itching or any other ocular discomfort were recorded. Tear film osmolarity was measured with TearLab (TearLab Osmolarity System, San Diego, CA, USA) on each eye before any other testing. Clinical examination with best corrected visual acuity (BCVA), converted to $\log$ MAR equivalent for statistical analysis, tonometry with iCare (Icare Finland Oy, Vantaa, Finland) and biomicroscopy with slit lamp examination $(\times 10$ and $\times$ 16 magnifications) and dilated funduscopy was performed. The tear film and Meibomian glands were assessed. Inflammatory or structural changes of the conjunctiva or cornea, including epithelial defects, corneal ulcers, scarring, corneal vascularization or conjunctivalization due to limbal stem cell deficiency, were noted. Conjunctival injection of the palpebral and bulbar conjunctiva was graded (grade 0: none, grade 1: mild/moderate, grade 2: severe) [14]. The iris, anterior chamber, lens and the posterior segment were examined and clinical findings indicating uveitis, cataract, glaucoma or retinal changes were noted. TBUT was measured after instillation of $2 \mu \mathrm{l}$ of $2 \%$ sodium fluorescein solution without preservatives onto the bulbar conjunctiva using a micropipette without inducing reflex tearing. The mean of three consecutive TBUTs was calculated. Corneal 
staining was assessed by slit lamp examination using cobalt blue illumination, 2.5-3 min after instillation of the fluorescein solution. The intensity of corneal fluorescein staining was graded according to the Oxford grading scale [20]. Schirmer I test (without anaesthesia) was performed for measurement of tear production. Sterile strips (TearFlo, Sigma Pharmaceuticals, North Liberty, IA, USA) were placed in the inferior fornix of the patients' eyes and removed after $5 \mathrm{~min}$. The length of wetting was measured in millimeters. The Ocular Surface Disease Index (OSDI) questionnaire (Norwegian translation) was used for assessment of the patient's subjective symptoms and graded as normal (0-12), mild (13-22), moderate (23-32) or severe $(\geq 33)$ [21]. Patients were classified as having oGVHD using the NIH criteria $[10,19]$ and as probable or definite oGVHD using the ICCGVH criteria [14].

Differentiating DED and oGVHD can be especially difficult in patients without systemic GVHD. Hence, only patients with known systemic chronic GVHD (cGVHD) (prior or current) were considered for the comparisons of oGVHD diagnosis by best clinical practice (BCP) and diagnosis by NIH or ICCGVHD criteria. Similar to previous studies, oGVHD diagnosis by BCP was defined as the gold standard [22, 23] and was based on overall clinical assessment and/or if at least two of the following five criteria were met: Schirmer I test $\leq 5 \mathrm{~mm}$ after $5 \mathrm{~min}, \mathrm{TBUT} \leq$ $5 \mathrm{~s}$, subjective ocular symptoms with $\mathrm{OSDI} \geq 13$, corneal staining $\geq$ grade 1 and conjunctival inflammation $\geq$ grade 1 [15]. Subsequently, diagnosis of oGVHD by ICCGVHD criteria or NIH criteria was compared to diagnosis by BCP.

Data regarding age at transplantation, date of transplantation, diagnosis, prior diagnosis of oGVHD, donor gender, donor type and any systemic treatment were obtained from medical records. Diagnosis of prior systemic acute GVHD (aGVHD) and cGVHD was based on chart review by a haematologist. As most of the study patients were transplanted before introduction of the NIH criteria, the criteria of Shulman et al. [24] were used for cGVHD grading.

\section{Statistical methods}

Statistical analyses were performed using SPSS version 22 (IBM SPSS, Armonk, NY, USA). Tests were two-sided, with significance level $p$-value $<0.05$. Patients included/ not included in the study were compared using the $\chi^{2}$ - and Mann-Whitney $U$-tests (tested for unequal variance, significance level adjusted for multiple comparisons using Bonferroni correction). Descriptive statistics for continuous variables (mean, SD, range) and frequencies/percentages of categorical variables were calculated. Agreement analyses were performed using linear $\kappa$-statistics $(\kappa)$. Sensitivity, specificity and negative/positive predictive values were calculated.
Association between the occurrence of DED and factors (patient gender, aGVHD, cGVHD, donor recipient gender mismatch, donor type, GVHD prophylaxis, malignancy, stem cell source and pre-transplantation conditioning regime) was investigated by univariate analyses $\left(\chi^{2}\right.$-test). Subsequently, multivariable logistic regression was performed with DED diagnosis as the dependent variable (Table 4). Of the 96 patients included in the data analysis, we excluded a total of three patients from the analysis due to lacking data regarding donor gender (one patient) and since the number of patients that received no conditioning was so few (only two patients). Before systematically selecting candidate variables for the statistical model, age at transplantation, age at eye examination, patient gender, the occurrence of any cGVHD (de novo or acute and chronic), the occurrence of aGVHD and steroid treatment, donor gender, donor type and conditioning were chosen to be included in the model due to clinical relevance. To investigate the effect of match or no match between patient gender and donor gender, we included the interaction between these two variables in the model. Furthermore, stepwise forward variable selection was then performed using likelihood ratio statistic as the selection criteria. We tested the inclusion of three additional variables (malignancy, GVHD prophylaxis and stem cell source). However, none of these were selected for the final model.

\section{Results}

\section{Patient characteristics}

The patient characteristics are summarized in Table 1. Of the 96 patients included in the analysis, 59 patients $(61 \%)$ had a history of systemic aGVHD or cGVHD and 11 patients (11\%) had known ocular involvement before the study examination.

\section{Ocular findings}

Fifty-two patients (54\%) were diagnosed with DED. The frequency distributions of patients with and without DED are shown in Table 2. Three patients (3\%) had posterior subcapsular cataract. Four patients had previously received total central nervous system (CNS) irradiation for CNS leukemia and all four had clinical DED. Of note, one of these irradiated patients also had microvascular retinopathy $(1 \%)$.

Of the 96 patients analysed, 85 patients $(89 \%)$ had good BCVA $(\log$ MAR $<0.1)$ in both eyes. Eleven patients $(11 \%)$ had reduced vision $(\log M A R>0.1)$. The causes for reduced vision were DED (four patients), cataract (two patients), 
Table 1 Patient characteristics ( $n=96$, data from medical records)

\begin{tabular}{|c|c|}
\hline Age at transplantation, mean \pm SD (years) & $\begin{array}{l}17.7 \pm 9.5 \text { (range } \\
0.3-30.0)\end{array}$ \\
\hline Age at eye examination, mean \pm SD (years) & $\begin{array}{l}34.4 \pm 11.1 \text { (range } \\
16.9-54.2 \text { ) }\end{array}$ \\
\hline $\begin{array}{l}\text { Time from transplantation to eye } \\
\text { examination, mean } \pm \mathrm{SD} \text { (years) }\end{array}$ & $\begin{array}{l}16.8 \pm 4.9 \text { (range } \\
6.0-26.1)\end{array}$ \\
\hline Recipient gender & $n(\%)$ \\
\hline Male & $44(46)$ \\
\hline Female & $52(54)$ \\
\hline Donor gender & $n(\%)$ \\
\hline Male & $49(51)$ \\
\hline Female & $46(48)$ \\
\hline Missing data & $1(1)$ \\
\hline Recipient/donor gender mismatch & $n(\%)$ \\
\hline Female recipient/ male donor & $23(24)$ \\
\hline Male recipient/ female donor & $18(19)$ \\
\hline Donor type & $n(\%)$ \\
\hline Sibling & $59(62)$ \\
\hline Other related & $7(7)$ \\
\hline Unrelated donor & $30(32)$ \\
\hline Diagnosis & $n(\%)$ \\
\hline Acute myeloid leukemia & $32(33)$ \\
\hline Acute lymphoblastic leukemia & $10(10)$ \\
\hline Other malignancies (CML/ MDS/ NHL) & $29(30)$ \\
\hline $\begin{array}{l}\text { Benign haematological diseases (anaemia/ } \\
\text { thalassaemia) }\end{array}$ & $17(18)$ \\
\hline Immunodeficiency/ metabolic disease & $8(8)$ \\
\hline Conditioning regime & $n(\%)$ \\
\hline Chemotherapy Bu/Cy & $73(76)$ \\
\hline Chemotherapy and antithymocyte globulin & $21(22)$ \\
\hline None & $2(2)$ \\
\hline GVHD prophylaxis & $n(\%)$ \\
\hline None & $1(1)$ \\
\hline СуA & $7(7)$ \\
\hline CyA + Mtx & $86(90)$ \\
\hline $\begin{array}{l}\text { CyA + MMF/ Sirolimus/ Tacrolimus/ Mtx/ } \\
\text { Other }\end{array}$ & $2(2)$ \\
\hline $\begin{array}{l}\text { History of radiotherapy affecting eye area } \\
\text { (incl. CNS radiotherapy) }\end{array}$ & $4(4)$ \\
\hline
\end{tabular}

$B u$ busulfan, $C y$ cyclophosphamide, $C y A$ cyclosporine, $C M L$ chronic myeloid leukemia, $M D S$ myelodysplastic syndrome, $M M F$ mycophenolate mofetil, Mtx methotrexate, $N H L$ non-Hodgkin's lymphoma

amblyopia (two patients), retinal vein occlusion (one patient) and unknown (two patients).

Characteristics of the tear function tests when used alone for diagnosis of DED or oGVHD (by NIH and ICCGVHD criteria) are shown in Table 3.
Table 2 Frequency distributions of patients with and without dry eye disease

\begin{tabular}{|c|c|c|c|}
\hline & $\begin{array}{l}\text { Patients with } \\
\text { DED }(n=52)\end{array}$ & $\begin{array}{l}\text { Patients without } \\
\text { DED }(n=44)\end{array}$ & $\begin{array}{l}\text { Total } \\
(n=96)\end{array}$ \\
\hline Gender (male/female) & $19 / 33$ & $25 / 19$ & $44 / 52$ \\
\hline \multicolumn{4}{|c|}{ Systemic GVHD after transplantation ${ }^{\mathrm{a}}$} \\
\hline No GVHD & 18 & 19 & 37 \\
\hline Only acute GVHD & 7 & 14 & 21 \\
\hline Only chronic GVHD & 10 & 2 & 12 \\
\hline $\begin{array}{l}\text { Acute and chronic } \\
\text { GVHD }\end{array}$ & 17 & 9 & 26 \\
\hline \multicolumn{4}{|c|}{ Systemic treatment of chronic GVHD } \\
\hline At any time & 23 & 9 & 31 \\
\hline $\begin{array}{l}\text { At time of eye } \\
\text { examination }\end{array}$ & 2 & 0 & 2 \\
\hline
\end{tabular}

$D E D$ dry eye disease, $G V H D$ graft-vs-host disease

${ }^{a}$ Based on chart review by haematologist

\section{Risk factors for DED}

Multivariable logistic regression revealed that a history of systemic cGVHD increased the risk of developing DED more than fourfold (odds ratio (OR) 4.40, confidence interval (CI) 1.33-14.56, $p=0.02$ ) (Table 4). Also, a trend was observed between donor type (non-sibling compared to sibling) and DED occurrence (OR 2.87, CI 0.91-9.11, $p=$ $0.07)$. Other factors included in the multivariable model (age at transplantation, age at eye examination, patient gender, donor gender, donor recipient gender relation, donor type, conditioning or aGVHD with/without steroid treatment) were not found to be statistically significant for DED occurrence.

\section{Comparison of diagnostic criteria}

In our study, 38 of 96 patients $(40 \%)$ had a history of systemic cGVHD. Diagnosis of oGVHD in these patients by BCP, by ICCGVHD criteria and NIH criteria, together with sensitivity, specificity and agreement, are shown in Table 5.

When applied to all 96 study patients, irrespective of systemic cGVHD status, 37 of the 52 DED patients (71\%) and 5 of the 44 patients without DED (11\%) were classified as oGVHD by NIH criteria. By ICCGVHD criteria, 23 of the DED patients (44\%) were classified as oGVHD (10 patients as definite oGVHD, 13 patients as probable oGVHD), whereas none of the patients without DED were classified as oGVHD. Agreement between oGVHD by NIH and ICCGVHD was moderate $(k=0.46)$. 


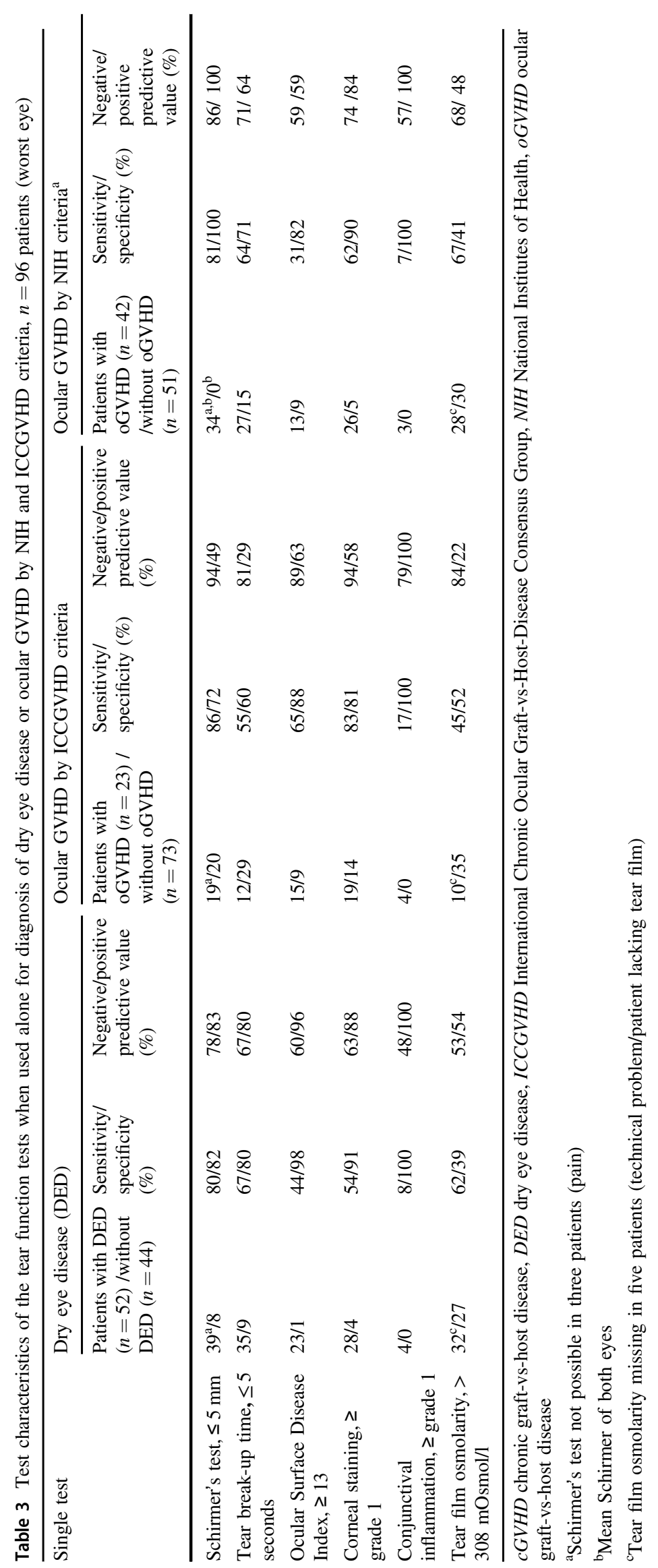


Table 4 Multivariable logistic regression with dry eye disease (DED) as the dependent variable

\begin{tabular}{|c|c|c|c|c|}
\hline \multirow[t]{2}{*}{ Variable } & \multirow[t]{2}{*}{ Odds ratio } & \multicolumn{2}{|c|}{$95 \% \mathrm{CI}$ for rdds ratio } & \multirow[t]{2}{*}{$p$-value ${ }^{\mathrm{a}}$} \\
\hline & & Lower & Upper & \\
\hline Age at eye examination & 1.05 & 0.95 & 1.16 & 0.38 \\
\hline Chronic GVHD vs no chronic GVHD & 4.40 & 1.33 & 14.56 & 0.02 \\
\hline Conditioning: other vs Busulfan/Cyclofosfamid & 0.43 & 0.13 & 1.39 & 0.16 \\
\hline Acute GVHD and steroid treatment & & & & 0.54 \\
\hline $\begin{array}{l}\text { No acute GVHD/no steroid treatment vs acute GVHD/ with } \\
\text { steroid treatment }\end{array}$ & 2.55 & 0.49 & 13.24 & 0.27 \\
\hline $\begin{array}{l}\text { Acute GVHD/no steroid treatment vs acute GVHD/with steroid } \\
\text { treatment }\end{array}$ & 1.74 & 0.39 & 7.81 & 0.47 \\
\hline Age at transplantation & 0.99 & 0.87 & 1.12 & 0.87 \\
\hline Patient gender/donor gender & & & & 0.28 \\
\hline $\mathrm{F} / \mathrm{M}$ vs $\mathrm{M} / \mathrm{F}$ & 3.85 & 0.77 & 19.2 & 0.10 \\
\hline $\mathrm{F} / \mathrm{F}$ vs $\mathrm{M} / \mathrm{F}$ & 1.54 & 0.36 & 6.52 & 0.56 \\
\hline $\mathrm{M} / \mathrm{M}$ vs $\mathrm{M} / \mathrm{F}$ & 1.05 & 0.25 & 4.37 & 0.95 \\
\hline Donor type: non-sibling vs sibling & 2.87 & 0.91 & 9.11 & 0.07 \\
\hline
\end{tabular}

${ }^{a}$ Statistical significance $p<0.05$

\section{Discussion}

Several studies on effects of allo-SCT and risk factors for development of oGVHD have been published [3-5, 25, 26]. However, a direct comparison of these is problematic due to differences in study design, population sizes, diagnostic criteria, treatment and follow-up times. To our knowledge, our study is the first to investigate the ocular effects of alloSCT for so long as a mean follow-up of 17 years after treatment in such a young group of patients (all patients $<$ 30 years at transplantation with mean age of about 18 years). Furthermore, in contrast to many other countries, the conditioning regimen prior to transplantation in Norway has usually been based only on myeloablative therapy without TBI. Hence, results of this study are especially relevant for the long term follow-up of young allo-SCT patients without TBI with respect to ocular findings.

In the general population, DED prevalence ranges from 6 to $33 \%$ [27-29]. In a Danish population aged 30-60 years, the prevalence of DED was found to be $11 \%$ [30]. Similarly, the Beaver Dam population-based study reported a prevalence rate of $14 \%$ in adults aged 21-49 years [28]. In our study, $54 \%$ of the patients were diagnosed with DED, which is notably higher than in the general population. Our findings are similar to previous studies, irrespective of TBI conditioning $[3,4,31]$. Thus, the occurrence of DED seems to be independent of TBI. On the other hand, a dose-related correlation between TBI conditioning and cataract development has been described, with cataract occurring in $20-100 \%$ of patients with TBI conditioning and in 3-19\% of patients without TBI treatment $[32,33]$. In line with these reports, we found a low occurrence of cataract (3\%).
Previously, it has been suggested that TBI may also contribute to posterior segment complications [34]. None of our study patients received TBI conditioning. However, four patients had a history of CNS radiotherapy, including one patient with microvascular retinopathy, which may be related to retinal changes induced by radiation [35].

We found that systemic cGVHD was associated with increased occurrence of DED. These findings are similar to others, despite differences in conditioning regimens $[4,26$, 31]. It is believed that the ocular surface changes seen in cGVHD are a consequence of destruction and fibrosis of the lacrimal gland [36], caused by extensive infiltration of inflammatory cells, including $\mathrm{T}$ cells, plasma cells, macrophages and fibroblasts [37]. Also, about half of patients with cGVHD have significant Meibomian gland dysfunction [3], which contributes to dry eye. In addition, both myeloablative therapy and TBI may cause host tissue damage [38], including damage to the lacrimal gland. Hence, both forms of conditioning may contribute to DED after allo-SCT.

In order to diagnose DED, we performed several functional tests, such as Schirmer's test, TBUT, corneal fluorescein staining, tear film osmolarity and OSDI, in addition to a comprehensive ocular examination. The reliability of such clinical tests and their correlation to DED severity has been questioned [13, 15, 17, 39-41], and their association to patient symptoms has been described as poor [21, 40]. In our study, as a single test, Schirmer's test had the highest sensitivity (80\%) among tests used to assist diagnosis of DED (Table 3). However, significant variations in reliability and repeatability of Schirmer's test have been reported previously [18, 41]. All our patients reported considerable discomfort during Schirmer's test. Along with factors such 
as light, humidity, room temperature and patient anxiety [40], this may influence reflex tearing, and may explain why one-fifth of the patients with DED (20\%) had normal Schirmer values. The OSDI questionnaire has been shown to be a valid and reliable tool for evaluation of DED severity [21], allowing rapid assessment of the patient's ocular symptoms and their impact on activities of daily life. Compared to clinical evaluation, the specificity of OSDI was an impressive $98 \%$. However, as more than half of the DED patients $(56 \%)$ did not experience ocular symptoms, the sensitivity was low (44\%). Hence, the OSDI was not reliable for diagnostic purposes in our patient population. Corneal fluorescein staining may be related to dry eye, but it is neither a specific nor a sensitive sign of DED [42]. Similarly, we found this test to have low sensitivity, with only half of the patients with DED (54\%) having corneal staining. Only $8 \%$ of the DED patients had conjunctival inflammation $\geq$ grade 1 , all with severe dry eye, indicating limited use of this parameter for diagnosis of DED in patients with mild/moderate disease. Although tear film osmolarity has been proposed as the single best test for assessment for DED severity [17], others have reported limited ability of tear film osmolarity to distinguish healthy subjects from patients with dry eye [43, 44]. The threshold value of $308 \mathrm{mOsm} / \mathrm{l}$ is considered to be a sensitive threshold for diagnosis of DED [45]. Using this threshold value in our study patients, almost one-third of the DED patients $(29 \%)$ were incorrectly classified as normal. Measurement of osmolarity may supplement a clinical diagnosis for grading of disease severity, but our data does not support its use as a stand-alone test for diagnostic purposes. In summary, our findings illustrate that no single test was adequate for diagnosing DED. Although the constellation of findings from functional tests may provide additional information to a clinical assessment, ocular evaluation by an ophthalmologist is advisable to ensure correct diagnosis of DED.

In our study, we noted a low incidence of oGVHD (11\%) diagnosed before our study examination. This observation may be due to the fact that patients were referred from various local hospitals in Norway for transplantation at Oslo University Hospital, which is a tertiary care centre. After transplantation, all patients were routinely followed up by a haematologist, with referral to ophthalmologist only when indicated. Thus, ocular complications may have been overseen, especially in mild cases.

The clinical spectrum of DED and oGVHD after alloSCT is overlapping and distinction may not always be possible. In lack of a consensus, clinical evaluation by an ophthalmologist is considered to be a historical gold standard $[9,15,16,23]$. Several proposals for diagnostic criteria have been made for oGVHD, including the NIH criteria and ICCGVHD criteria [7, 14, 19], and the observed occurrence 
of oGVHD is obviously dependent on which diagnostic criteria are used $[10,14,19]$. The ICCGVH criteria considers the presence or absence of chronic systemic GVHD, whereas, according to the NIH criteria, new onset of KCS by slit lamp exam with low Schirmer's test is sufficient for the diagnosis of ocular cGVHD for the purpose of clinical oGVHD trials $[9,15,16]$. The emphasis on Schirmer's test for oGVHD diagnosis by NIH criteria is debated [7, 14, 15]. Adversaries advocate that high rates of false positives and negatives render Schirmer's test a poor diagnostic test for oGVHD [3, 15]. Hence, although the NIH criteria may be a valuable tool for grading systemic cGVHD by transplant physicians, they are inadequate for accurate diagnosis of oGVHD. The ICCGVHD criteria have recently been validated in chart review study including only patients with suspected or confirmed chronic oGVHD [23], but to our knowledge, there is no study comparing these criteria to the well-known NIH criteria. Due to clinical overlap between DED and oGVHD warranting a more robust analysis, we initially considered only patients with a history of systemic cGVHD when diagnostic criteria were compared with BCP as the gold standard (Table 5). We found substantial agreement between diagnosis by BCP and NIH and between BCP and ICCGVD criteria $(\kappa=0.68$ and $\kappa=0.67$, respectively), with good sensitivity and specificity. Furthermore, there was substantial agreement between diagnosis by NIH and ICCGVHD criteria $(\kappa=0.70)$. On the other hand, when the criteria were applied to all the study patients, more than two-thirds $(71 \%)$ of the DED patients were classified as having oGVHD by NIH criteria, whereas by ICCGVD criteria, less than half $(44 \%)$ had definite or probable oGVHD. Also, only moderate agreement was observed between the two sets of diagnostic criteria $(\kappa=0.46)$. This discrepancy is not unexpected, as the NIH criteria are based on Schirmer's test, whereas the ICCGVHD criteria include the patient's systemic cGVHD status and also three additional parameters. Hence, the more stringent ICCGVHD criteria seem to better differentiate DED from oGVHD.

Limitations of our study include lack of pretransplantation baseline ocular examination, wide range in time from transplantation to ocular examination and lack of a control group matched for age and gender. However, in our study, the mean age at transplantation was 34.4 years and ocular findings such as DED are not so frequent in healthy persons in this age group. Also, set time-point examinations would allow inferences to be made about time from transplantation to onset of ocular symptoms or whether ocular symptoms occurred before or after onset of systemic signs. Since age $\leq 30$ years was an inclusion criteria, the results of our study may not be applicable to patients transplanted at older age.

In conclusion, half of the patients treated with allo-SCT without TBI were diagnosed with DED by an ophthalmologist more than 5 years after transplantation. Systemic cGVHD increased the risk of DED more than fourfold. As expected, we found a very low occurrence of radiation-related complications such as cataract and posterior segment involvement. Clinically, Schirmer's test caused discomfort to the patient and was frequently in disaccord with other tests. In comparison, corneal staining, TBUT and assessment of patient symptoms with OSDI questionnaire added useful information to the overall ocular evaluation. Our comparison of diagnostic criteria suggests that the more stringent ICCGVHD criteria can better differentiate DED from oGVHD after allo-SCT as compared with the NIH criteria. Larger studies are warranted for further validation and comparison of diagnostic criteria. As a significant proportion of allo-SCT patients have ocular complications many years after transplantation, evaluation by an ophthalmologist should be included in the management of these patients.

Acknowledgements The research leading to these results has received funding from the Norwegian Extra Foundation for Health and Rehabilitation project number 2013-2-230 and the South-Eastern Norway Regional Health Authority, Norway grant number 2015084. Financial Support: The study was partially financed by the Norwegian Extra Foundation for Health and Rehabilitation project no. 2013-2-230 and the South-Eastern Norway Regional Health Authority, Norway grant number 2015084. The funding organizations had no role in the design or conduct of this research.

\section{Compliance with ethical standards}

Conflict of interest The authors declare that they have no conflict of interest.

Open Access This article is licensed under a Creative Commons Attribution-NonCommercial-NoDerivatives 4.0 International License, which permits any non-commercial use, sharing, distribution and reproduction in any medium or format, as long as you give appropriate credit to the original author(s) and the source, and provide a link to the Creative Commons license. You do not have permission under this license to share adapted material derived from this article or parts of it. The images or other third party material in this article are included in the article's Creative Commons license, unless indicated otherwise in a credit line to the material. If material is not included in the article's Creative Commons license and your intended use is not permitted by statutory regulation or exceeds the permitted use, you will need to obtain permission directly from the copyright holder. To view a copy of this license, http://creativecommons.org/licenses/by-nc-nd/4.0/.

\section{References}

1. Lee SJ, Flowers ME. Recognizing and managing chronic graftversus-host disease. Hematology. American Society of Hematology Education Program 2008:134-141. (e-pub ahead of print 2008/12/17; https://doi.org/10.1097/ICO.0b013e3181ca321c).

2. Tichelli A, Duell T, Weiss M, Socie G, Ljungman P, Cohen A, et al. Late-onset keratoconjunctivitis sicca syndrome after bone marrow transplantation: incidence and risk factors. European 
Group or Blood and Marrow Transplantation (EBMT) Working Party on Late Effects. Bone Marrow Transplant. 1996;17:1105-11.

3. Ogawa Y, Okamoto S, Wakui M, Watanabe R, Yamada M, Yoshino M, et al. Dry eye after haematopoietic stem cell transplantation. Br J Ophthalmol. 1999;83:1125-30.

4. Westeneng AC, Hettinga Y, Lokhorst H, Verdonck L, van Dorp S, Rothova A. Ocular graft-versus-host disease after allogeneic stem cell transplantation. Cornea. 2010;29:758-63. https://doi.org/10. 1097/ICO.0b013e3181ca321c.

5. Kerty E, Vigander K, Flage T, Brinch L. Ocular findings in allogeneic stem cell transplantation without total body irradiation. Ophthalmology. 1999;106:1334-8. https://doi.org/10.1016/ S0161-6420(99)00720-4.

6. Nassiri N, Eslani M, Panahi N, Mehravaran S, Ziaei A, Djalilian AR. Ocular graft versus host disease following allogeneic stem cell transplantation: a review of current knowledge and recommendations. J Ophthal Vis Res. 2013;8:351-8.

7. Dietrich-Ntoukas T, Cursiefen C, Westekemper H, Eberwein $\mathrm{P}$, Reinhard T, Bertz H, et al. Diagnosis and treatment of ocular chronic graft-versus-host disease: report from the GermanAustrian-Swiss Consensus Conference on Clinical Practice in chronic GVHD. Cornea. 2012;31:299-310. https://doi.org/10. 1097/ICO.0b013e318226bf97.

8. Lemp MA. Report of the National Eye Institute/Industry workshop on Clinical Trials in Dry Eyes. CLAO J: Off Publ Contact Lens Assoc Ophthalmol, Inc. 1995;21:221-32. e-pub ahead of print 1995/10/01.

9. Baudouin C, Aragona P, Van Setten G, Rolando M, Irkeç M, Benítez del Castillo J, et al. Diagnosing the severity of dry eye: a clear and practical algorithm. Br J Ophthalmol. 2014;98:1168-76. https://doi.org/10.1136/bjophthalmol-2013-304619.

10. Jagasia MH, Greinix HT, Arora M, Williams KM, Wolff D, Cowen EW, et al. National Institutes of Health Consensus Development Project on Criteria for Clinical Trials in Chronic Graft-versus-Host Disease: I. The 2014 Diagnosis and Staging Working Group report. Biol Blood Marrow Transplant: J Am Soc Blood Marrow Transplant. 2015;21:389-401. e381. https://doi. org/10.1016/j.bbmt.2014.12.001.

11. Kim S. Ocular graft versus host disease. In: Krachmer JH, Mannis MJ, Holland EJ (eds). Cornea. Elsevier/Mosby: Philadelphia, 2004, pp 879-85.

12. Coskuncan NM, Jabs DA, Dunn JP, Haller JA, Green WR, Vogelsang GB, et al. The eye in bone marrow transplantation. VI. Retinal complications. Arch Ophthalmol (Chic, Ill: 1960). 1994;112:372-9. e-pub ahead of print 1994/03/01.

13. Anonymous. The definition and classification of dry eye disease: report of the Definition and Classification Subcommittee of the International Dry Eye WorkShop (2007). Ocul Surf. 2007;5:75-92. e-pub ahead of print 2007/05/18.

14. Ogawa Y, Kim SK, Dana R, Clayton J, Jain S, Rosenblatt MI, et al. International Chronic Ocular Graft-vs-Host-Disease (GVHD) Consensus Group: proposed diagnostic criteria for chronic GVHD (Part I). Sci Rep. 2013;3:3419 https://doi.org/ $10.5791038 /$ srep03419.

15. Jacobs R, Tran U, Chen H, Kassim A, Engelhardt BG, Greer JP, et al. Prevalence and risk factors associated with development of ocular GVHD defined by NIH consensus criteria. Bone Marrow Transplant. 2012;47:1470-3. https://doi.org/10.1038/bmt. 5842012.56.

16. Anonymous. Methodologies to diagnose and monitor dry eye disease: report of the Diagnostic Methodology Subcommittee of the International Dry Eye WorkShop (2007). Ocul Surf. 2007;5:108-52.

17. Sullivan BD, Whitmer D, Nichols KK, Tomlinson A, Foulks GN, Geerling $G$, et al. An objective approach to dry eye disease severity. Invest Ophthalmol Vis Sci. 2010;51:6125-30. e-pub 592ahead of print 2010/07/16, 10.1167/iovs.10-5390.

18. Serin D, Karsloglu S, Kyan A, Alagoz G. A simple approach to the repeatability of the Schirmer test Without anesthesia: eyes open or closed? Cornea. 2007;26:903-6.

19. Filipovich AH, Weisdorf D, Pavletic S, Socie G, Wingard JR, Lee SJ, et al. National Institutes of Health consensus development project on criteria for clinical trials in chronic graft-versus-host disease: I. Diagnosis and staging working group report. Biol Blood Marrow Transplant. 2005;11:945-56. https://doi.org/10. 1016/j.bbmt.2005.09.004.

20. Bron AJ, Evans VE, Smith JA. Grading of corneal and conjunctival staining in the context of other dry eye tests. Cornea. 2003;22:640-50.

21. Schiffman RM, Christianson MD, Jacobsen G, Hirsch JD, Reis BL. Reliability and validity of the Ocular Surface Disease Index. Arch Ophthalmol (Chic, Ill: 1960). 2000;118:615-21.

22. Tatematsu Y, Ogawa Y, Abe T, Kamoi M, Uchino M, Saijo-Ban $\mathrm{Y}$, et al. Grading criteria for chronic ocular graft-versus-host disease: comparing the NIH eye score, Japanese dry eye score, DEWS 2007 ssore. Sci Rep. 2014;4:6680.

23. Rapoport Y, Freeman T, Koyama T, Engelhardt BG, Jagasia M, Savani BN, et al. Validation of International Chronic Ocular Graft-Versus-Host Disease (GVHD) Group Diagnostic Criteria as a Chronic Ocular GVHD-Specific Metric. Cornea. 2017;36:258-63.

24. Shulman HM, Sullivan KM, Weiden PL, McDonald GB, Striker GE, Sale GE, et al. Chronic graft-versus-host syndrome in man. A long-term clinicopathologic study of 20 Seattle patients. Am J Med. 1980;69:204-17.

25. Tabbara KF, Al-Ghamdi A, Al-Mohareb F, Ayas M, Chaudhri N, Al-Sharif F, et al. Ocular findings after allogeneic hematopoietic stem cell transplantation. Ophthalmology. 2009;116:1624-9. https://doi.org/10.1016/j.ophtha.2009.04.054.

26. Wang JC, Teichman JC, Mustafa M, O'Donnell H, Broady R, Yeung SN. Risk factors for the development of ocular graftversus-host disease (GVHD) dry eye syndrome in patients with chronic GVHD. Br J Ophthalmol. 2015;99:1514-8.

27. Shimmura S, Shimazaki J, Tsubota K. Results of a populationbased questionnaire on the symptoms and lifestyles associated with dry eye. Cornea. 1999;18:408-11.

28. Paulsen AJ, Cruickshanks KJ, Fischer ME, Huang GH, Klein BE, Klein R, et al. Dry eye in the beaver dam offspring study: prevalence, risk factors, and health-related quality of life. Am J Ophthalmol. 2014;157:799-806.

29. Schaumberg DA, Sullivan DA, Buring JE, Dana MR. Prevalence of dry eye syndrome among US women. Am J Ophthalmol. 2003;136:318-26.

30. Bjerrum KB. Keratoconjunctivitis sicca and primary Sjogren's syndrome in a Danish population aged 30-60 years. Acta Ophthalmol Scand. 1997;75:281-6.

31. Khan R, Nair S, Seth T, Mishra P, Mahapatra M, Agarwal T, et al. Ocular graft versus host disease in allogenic haematopoetic stem cell transplantation in a tertiary care centre in India. Indian J Med Res. 2015;142:543-8.

32. Tichelli A, Gratwohl A, Egger T, Roth J, Prunte A, Nissen C, et al. Cataract formation after bone marrow transplantation. Ann Intern Med. 1993;119:1175-80.

33. Benyunes MC, Sullivan KM, Deeg HJ, Mori M, Meyer W, Fisher $\mathrm{L}$, et al. Cataracts after bone marrow transplantation: long-term follow-up of adults treated with fractionated total body irradiation. Int J Radiat Oncol Biol Phys. 1995;32:661-70.

34. Majhail NS, Rizzo JD, Lee SJ, Aljurf M, Atsuta Y, Bonfim C, et al. Recommended screening and preventive practices for longterm survivors after hematopoietic cell transplantation. Rev Bras De Hematol e Hemoter. 2012;34:109-33. 
35. Gordon KB, Char DH, Sagerman RH. Late effects of radiation on the eye and ocular adnexa. Int J Radiat Oncol Biol Phys. 1995;31:1123-39.

36. Jack MK, Jack GM, Sale GE, Shulman HM, Sullivan KM. Ocular manifestations of graft-v-host disease. Arch Ophthalmol (Chic, Ill: 1960). 1983;101:1080-4.

37. Ogawa Y, Yamazaki K, Kuwana M, Mashima Y, Nakamura Y, Ishida $\mathrm{S}$, et al. A significant role of stromal fibroblasts in rapidly progressive dry eye in patients with chronic GVHD. Invest Ophthalmol Vis Sci. 2001;42:111-9.

38. Hessen M, Akpek EK. Ocular graft-versus-host disease. Curr Opin Allergy Clin Immunol. 2012;12:540-7.

39. Inamoto $\mathrm{Y}$, Chai $\mathrm{X}$, Kurland $\mathrm{BF}$, Cutler $\mathrm{C}$, Flowers ME, Palmer $\mathrm{JM}$, et al. Validation of measurement scales in ocular graft-versushost disease. Ophthalmology. 2012;119:487-93.

40. Nichols KK, Nichols JJ, Mitchell GL. The lack of association between signs and symptoms in patients with dry eye disease. Cornea. 2004;23:762-70.
41. Nichols KK, Mitchell GL, Zadnik K. The repeatability of clinical measurements of dry eye. Cornea. 2004;23:272-85.

42. Savini G, Prabhawasat P, Kojima T, Grueterich M, Espana E, Goto E. The challenge of dry eye diagnosis. Clin Ophthalmol (Auckl, N Z). 2008;2:31-55.

43. Messmer EM, Bulgen M, Kampik A. Hyperosmolarity of the tear film in dry eye syndrome. Dev Ophthalmol. 2010;45:129-38. https://doi.org/10.1159/000315026.

44. Szalai E, Berta A, Szekanecz Z, Szucs G, Modis L Jr.. Evaluation of tear osmolarity in non-Sjogren and Sjogren syndrome dry eye patients with the TearLab system. Cornea. 2012;31:867-71. https://doi.org/10.1097/ICO.0b013e3182532047.

45. Lemp MA, Bron AJ, Baudouin C, Benitez Del Castillo JM, Geffen D, Tauber J, et al. Tear osmolarity in the diagnosis and management of dry eye disease. Am J Ophthalmol. 2011;151:792 https://doi.org/10.1016/j.ajo.2010.10.032. 